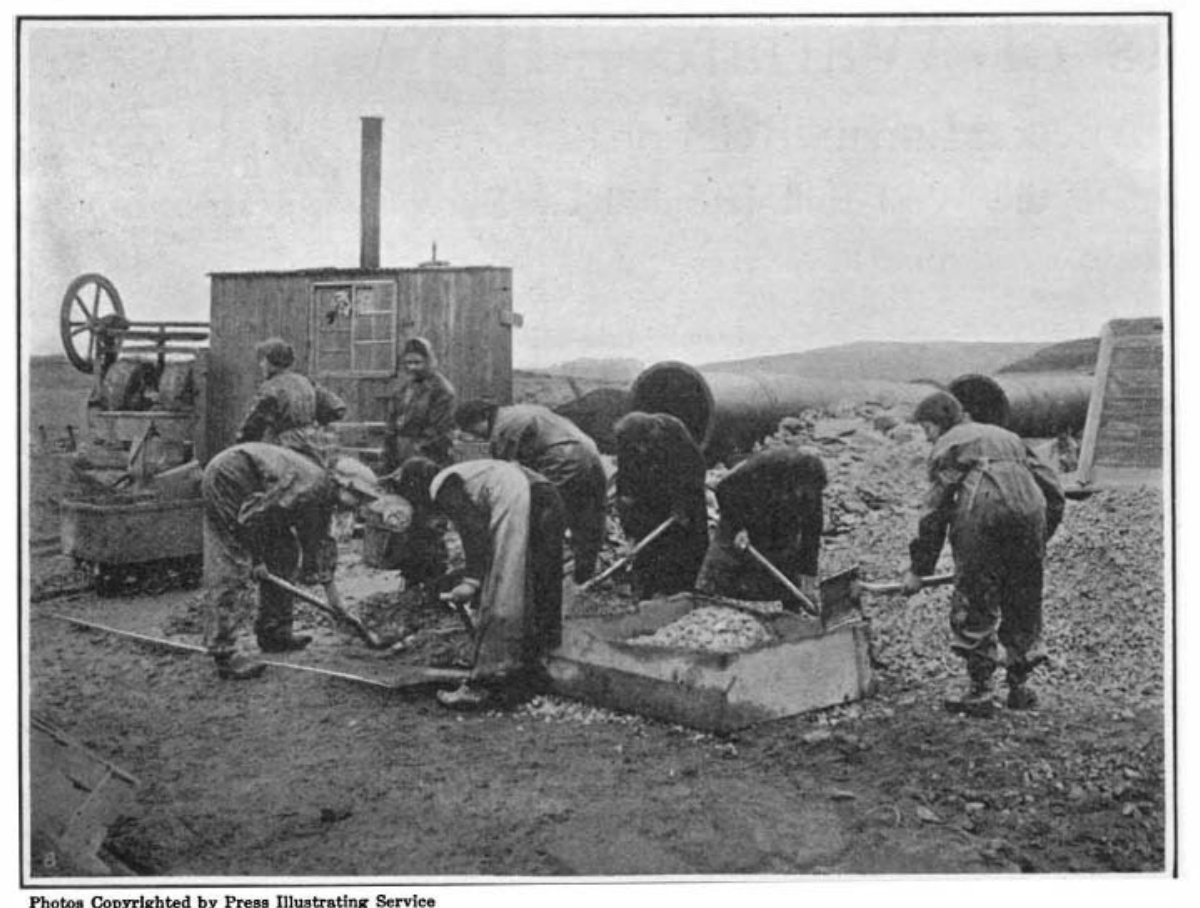

Mixing concrete by hand for engineering work

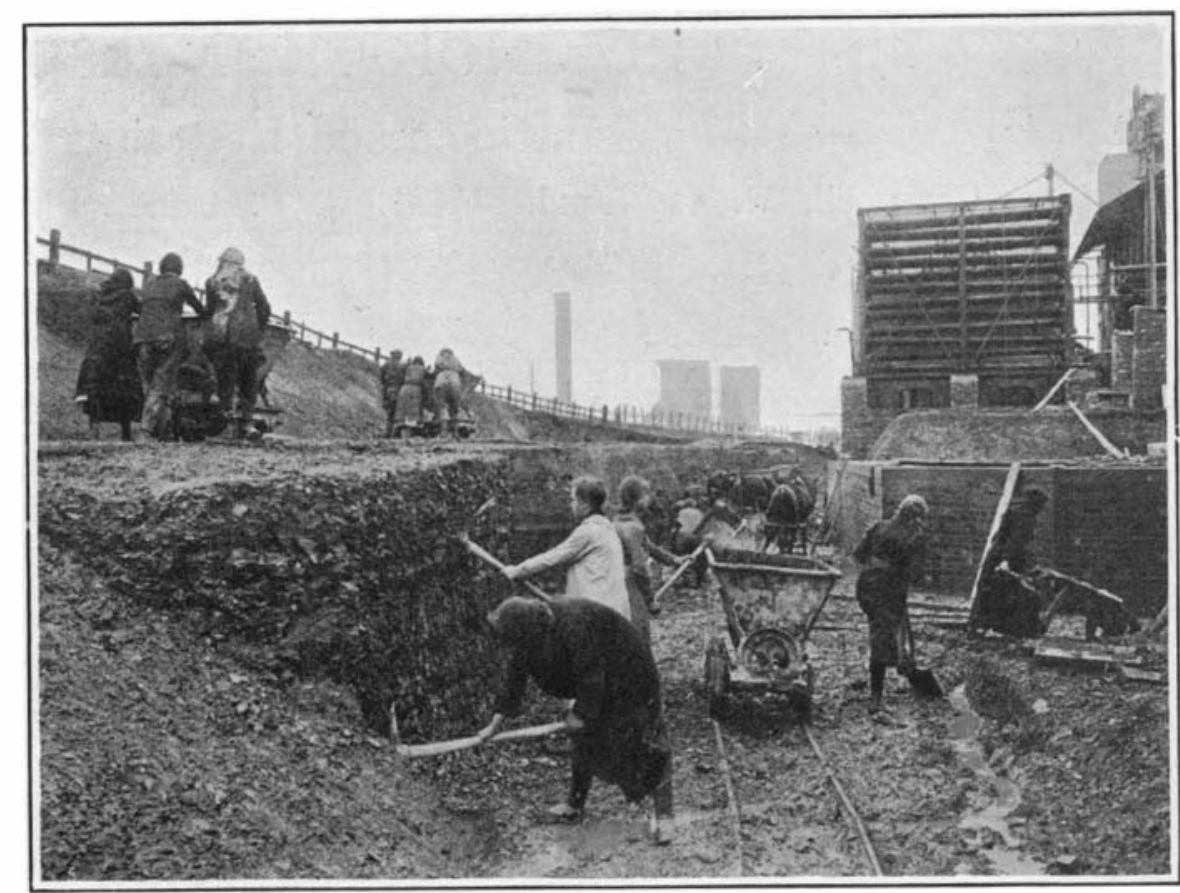

Excavating preparatory to building operations
Evidence of Matter in Space Obstructing the Passage of Light*

By W. W. Campbell

OđR photographic telescopes are confirming William Herschel's observation that the large and formless nebulæ are in or are bordered by regions of sky showing fewer stars than abound in the surrounding regions. The bright stars in the Pleiades, those really belonging to the cluster, are numerous, but within the cluster as we see it, and in a considerable area of the adjacent sky, the faint stars are markedly scarcer than in the areas farther away. Barnard has found that the sky in the region around the Pleiades group is possessed of much nebulosity. It is a natural question, are the faint stars scarce because the nebulosity there existing has not yet condensed into stellar forms? This may be true in part, but we shall find much more probable the view that the faint stars are deficient in numbers because the nebular materials, at a certain distance away from us, are obstructing the light of the faint stars that are farther away from us than the nebula is. A similar deficiency of faint stars exists within the great nebula of Orion, and likewise in the adjacent areas, where Mr. William H. Pickering has found a very large part of the constellation of Orion to be covered with faint nebulosity. There are so many regions in and near the galaxy where these relationships exist (that is, a presence of nebulosity and a scarcity of faint stars) that we can not doubt their significance. The faint stars are relatively scarce chiefly because the nebular materials cut off the light of the more distant stars. This explanation is reached by several lines of evidence, but we take time to present only one.

It is established by modern astronomy that the individual stars are in rapid motion. The speeds of the naked eye stars average about sixteen miles per second. The distant fainter stars, so far as they have been observed, are also traveling rapidly. There is a tendency to favor certain directions of motion, and the stars in certain small groups are keeping company through space but a large share of stellar motion is at random. There are stars traveling in all directions. We have not the direct evidence as to the motions of the faint stars in the direct evidence as to the motions of the faint stars in the to suspect that their characteristic motions are unique. We can see no escape from the condition that all the stars are in motion. Under these circumstances we are unable to explain how within a great volume of space that is rich in stars there can be a smaller, but still enormous volume of space, nearly free of stars. Their random motions should distribute them more uniformly than we observe to be the case. Let us illustrate by the celebrated black holes, the so-called "coal sacks," in the constellation of Sagittarius. In a region where the stars are especially plentiful are two small areas all but empty of visible stars. How can such great "holes" throug the stellar aystem be surrounded by a plentitude of stars moving more or less at random so as to give a high density of star distribution right up to the sharply defined edge of the holes, and yet leave the holes empty of stars? With the help of all astronomical experience we can no explain the phenomena by the absence of stars. I think we must assume, with Barnard and others, that the stars are actually there, and that they are invisible because invisible materials between us and the stars are absorbing or occulting the light which the stars are trying to send us. Much of the interesting structure in *From a presidental address to the Am Ass. for the Advance-
ment of sclence the Milky Way is probably due in a like manner to obstruction by materials lying between us and the great clouds of stars. It is characteristic of the galactic structure that where luminous nebula seem to reduce the numbers of faint stars visible, the reduction in numbers of stars extends also far out beyond the limits of visible nebulosity, and we can scarcely resist the conclusion that invisible extensions of the luminous nebular fabric exist as obstructing agents.

There are many other lines of evidence in support of the hypothesis that invisible matter exists in abundance within the stellar system:

1. Newcomb and Kelvin, working independently and on the assumption that the motions of the stars ar generated by gravitational attractions originating within the stellar system, were unable to account for the high observed velocities of stars, except on the hypothesis that the visible stars contain only a small fraction of the matter existing in the system; the greater part of the attracting material in the stellar system being nonluminous, or at least invisible.

2. There must be an enormous amount of come material distributed throughout space. The astronomers discover only a negligibly small proportion of the comets which pass near the center of our solar system. May not comet materials exist also in abundance in the systems of the other stars?

3. Students of meteors have established that the separate little bodies which collide with the earth' atmosphere and are responsible for the so-called shooting stars are stupendous in numbers. It hss been estimated that as many as twenty or thirty millions of such bodies collide with the earth every 24 hours. How incomparably and inconceivably greater must be the total number in our solar system. The power of these count less particles to obstruct the passage of light is not negligible. There may be as many such particles on th average around the other stars in our stellar system.

4. The so-called new stars, otherwise known as temporary stars, afford interesting evidence on this point. These are stars which suddenly flash out at points where previously no stars were known to exist; or, in a few cases, where a faint existing star has in a few days become immensely brighter. Twenty-nine such stars have been observed in the past three centuries, 19 of them since 1886 when the photographic dry plate was applied systematically to the mapping of the heavens, and 15 of the 19 are to the credit of the Harvard observers. This is an average of one new star in less than two years in the last three decades; and, as some of the fainter new stars undoubtedly come and go unseen, it is evident that they are by no means rare objects. Now all of th temporary stars except five appeared in the Milky Way, and four of the five exceptions are worthy of note. Two of the five appeared in well-known nebulæ; another wa located close to the edge of a spiral nebula, and quite probably in a faint outlying part of it; a fourth wa observed to have a nebulous halo about it; and the fift was but meagerly and imperfectly observed. Keeping the story as short as possible, a temporary star is seemingly best explained on the theory that a dark or relatively dark star traveling rapidly through space has met with resistance, such as a great nebula or cloud of particles would afford. While passing through the cloud the star is in effect bombarded at high velocity by the resisting materials. The surface strata become heated, and the luminosity of the star increases rapidly. The new star of February, 1901, in Perseus afforded interest-
ing testimony. Wolf, at Heidelberg, photographed in
August an irregular nebulous object near the star. Ritchey's photograph of September showed extensive areas of nebulosity in all directions from the star. In October Perrine and Ritchey discovered that the nebular structure had apparently moved outward from the star. Going back to a March photograph, taken for a different purpose, Perrine found recorded upon it an irregular ring of nebulosity closely surrounding the star which was not visible on later photographs. The region seemed to be full of nebulosity not visible to us under normal conditions. The rushing of the dark star into and through this resisting medium made the star the brightest one in the northern sky for several days. The great wave of light going out from the star when at this maximum brightness traveled far enough in five weeks to fall upon non-luminous materials and made a ring of nebulosity visible by reflection. Continuing its progress, with a speed of 186,000 miles per second, the wave of light illuminated the material which Wolf photographed far away from the star in August, the material which Ritchy photographed still farther away in September, and the still more distant materials which Perrine and Ritchey photographed in October, November and in later months. We were able to see this material only as the very strong wave of light which left the star at maximum brightness made the material luminous in passing.

We can scarcely doubt, in view of all these facts, that there is a stupendous amount of obstructing material scattered throughout our stellar system. This may eventually be condensed into stars; but the point I wish to emphasize is that the material is there, and is evidently obstructing the passage of light. The efficiency of the obstructing material is, no doubt, the greater in the long dimensions of the system; in the direction of the Milky Way.

\section{Protection against Noise and Vibration}

THE problem resolves itself to protection against "air noises" and against "ground noises." Protection against air noises may be obtained by air-tight enclosures and by measures preventing bending oscillations in (or the propagation of oscillations from) the "soundproof" walls. If a body is able to communicate its oscillations to the ground, we have to consider "ground noises" which, according to the nature of the vibrations and materials concerned, may be propagated by compression waves, thrust waves, expansion waves, surface waves, or bending waves. Light and loosely packed material damps ground noises by propagating them with reduced velocity and intensity. Where applicable, narrow sirgaps round a machine are effective against surface waves. Otherwise, insulating material should be used which withstands large alterations in thickness without loss of elasticity. Curves in the original show the amplitude of vibrations set up in a floor by a motor running at various speeds, with and without the use of a buffer mat. In this particular case there were amplitude maxima at 1,350 , 1,700 and 2,550 r.p.m., without the mat. With the latter, the amplitudes of these three maxima were changed from $0.025,0.091$ and $0.235 \mathrm{~mm}$. to $0.031,0.091$ and $0.11 \mathrm{~mm}$. respectively and the speeds at which they occurred were reduced to $1,340,1,600$ and 2,100 r.p.m. The amplitude of the principal vibration within this speed-range was much reduced by the use of a damping mat, but the change in position of the vibration-speed curve was such that at 2,500 r.p.m. the mat reduced the amplitude of vibration to one-quarter.- Note in Science Abstracts on an article by $\mathrm{K}$. Henck in Die Naturwissenschaften. 